\title{
Numerical and experimental investigation of the stability of a drop in a single-axis acoustic levitator $\odot$
}

Cite as: Phys. Fluids 31, 117101 (2019); https://doi.org/10.1063/1.5121728

Submitted: 27 July 2019 . Accepted: 20 October 2019 . Published Online: 04 November 2019

Marco A. B. Andrade (D), and Asier Marzo (D)

\section{COLLECTIONS}

Paper published as part of the special topic on Drops and Flows in Acoustic Levitation Note: This paper is part of the Special Topic on Drops and Flows in Acoustic Levitation.

F This paper was selected as Featured

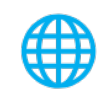

\section{ARTICLES YOU MAY BE INTERESTED IN}

Review of transport processes and particle self-assembly in acoustically levitated nanofluid droplets

Physics of Fluids 31, 112102 (2019); https://doi.org/10.1063/1.5125059

Internal flow during mixing induced in acoustically levitated droplets by mode oscillations Physics of Fluids 31, 112101 (2019); https://doi.org/10.1063/1.5124988

Beyond the Langevin horn: Transducer arrays for the acoustic levitation of liquid drops Physics of Fluids 31, 101301 (2019); https://doi.org/10.1063/1.5117335

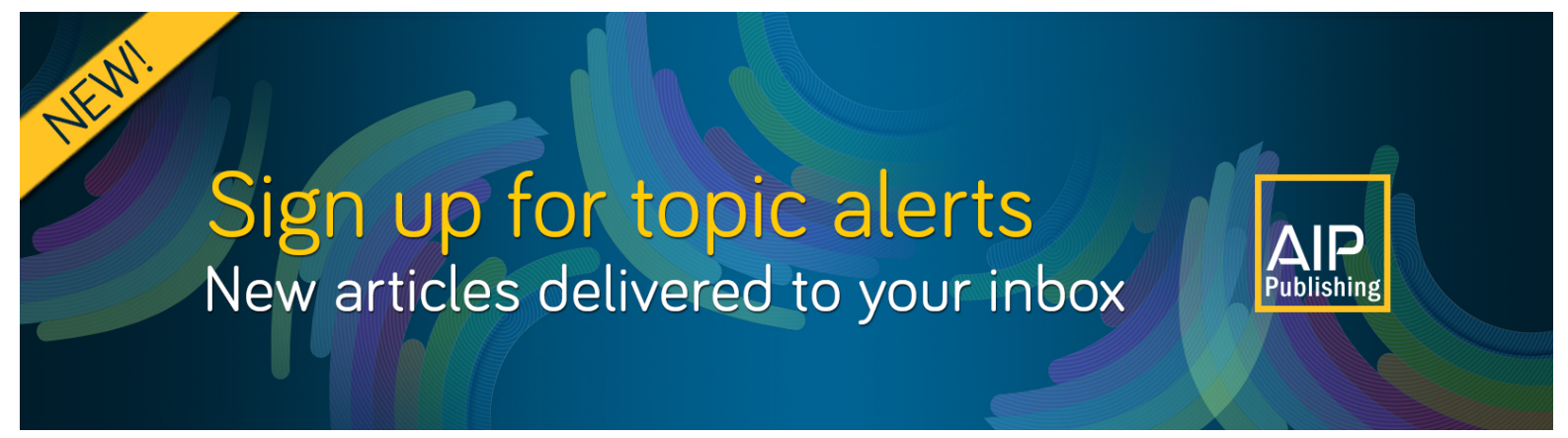




\title{
Numerical and experimental investigation of the stability of a drop in a single-axis acoustic levitator
}

\author{
Cite as: Phys. Fluids 31, 117101 (2019); doi: 10.1063/1.5121728 \\ Submitted: 27 July 2019 - Accepted: 20 October 2019 • \\ Published Online: 4 November 2019
}

\author{
Marco A. B. Andrade ${ }^{1, a)}$ (D) and Asier Marzo \\ AFFILIATIONS \\ ${ }^{1}$ Institute of Physics, University of São Paulo, São Paulo 05508-090, Brazil \\ ${ }^{2}$ Computer Science, Public University of Navarre, Pamplona 31006, Navarre, Spain
}

Note: This paper is part of the Special Topic on Drops and Flows in Acoustic Levitation.

a) Author to whom correspondence should be addressed: marcobrizzotti@gmail.com

\begin{abstract}
Acoustic levitation can be employed to hold liquid drops in midair, enabling novel applications in X-ray scattering of proteins, amorphous crystallization of solutions, or contactless mixing. Multiple studies have characterized the physical behavior of a levitated drop inside an acoustic field. Here, we present a numerical and experimental study on the acoustic levitation of water drops in a single-axis acoustic levitator consisting of an ultrasonic transducer and an opposing reflector. Instead of modeling an abstract incident acoustic field, our model considers the shape of the drop as well as the real geometry of the levitator. We also use a high-speed camera to observe the disintegration and the undesired oscillations of the drops. Our results show that the insertion of a drop in the levitator provokes a shift in its resonant frequency that depends on the shape of the drop. Second, the levitation behavior depends on whether the levitator operates slightly below or above the resonance. Third, if the levitator is driven above the resonant frequency, it is possible to levitate with more strength and avoid disintegration of the drop. This research provides an insight on how to achieve more stable experiments that avoid the bursting and undesired oscillations of the levitated sample. We hope that it will facilitate numerous experiments involving acoustically levitated liquid drops.
\end{abstract}

Published under license by AIP Publishing. https://doi.org/10.1063/1.5121728

\section{INTRODUCTION}

Contactless manipulation of liquid drops is of great interest in disciplines such as analytical chemistry, ${ }^{1,2}$ pharmacy, ${ }^{3}$ or biology. ${ }^{4}$ Different techniques have been used for manipulating small drops, including electric, ${ }^{5-8}$ magnetic, ${ }^{9-11}$ thermocapillary, ${ }^{12,13}$ and acoustic waves. ${ }^{14,15}$ Among different techniques, contactless manipulation based on acoustic waves has the main advantage of being flexible, allowing the manipulation of different materials inside liquids or in midair. It can also be used for manipulating samples with sizes ranging from less than $1 \mu \mathrm{m}$ to several millimeters.

The use of acoustic waves to hold objects in midair is called acoustic levitation, ${ }^{17-20}$ and it uses the phenomenon of the acoustic radiation force ${ }^{21}$ to counteract the gravitational force acting on the levitated object. There are different methods to levitate objects acoustically, with the most common being the trapping of small objects at the pressure nodes of a standing wave. ${ }^{22,23}$ This approach allows the suspension of objects and controlling of their position in one, ${ }^{24-26}$ two, ${ }^{14,27}$ and three ${ }^{28,29}$ dimensions. Furthermore, multiple objects can be manipulated independently.

Acoustic levitation can also be combined with spectroscopic methods to characterize levitating liquid samples, ${ }^{1,31,32}$ e.g., it can be combined with X-ray diffraction for determining the structure of proteins ${ }^{33}$ or it can be applied for monitoring the crystallization of levitated substances. ${ }^{34}$ Acoustic levitation can also be applied for measuring the surface tension of liquid drops ${ }^{35,36}$ and for studying the evaporation of liquids. ${ }^{37,38}$ In general, acoustic levitation is an important contactless manipulation method for the transportation and merging of liquid substances in midair.

Reliable methods for acoustically manipulating drops require a good understanding of the stability of levitating drops under the high intensity acoustic fields employed for levitation. This is not a 
trivial problem since the acoustic field has to be strong enough to counteract the gravitational force but cannot exceed a certain limit that would cause the disintegration of the drops. The determination of the equilibrium shape of levitating drops, ${ }^{41-47}$ its disintegration, ${ }^{43,48-50}$ and the dynamics ${ }^{51-54}$ of levitating drops have been described in numerous studies. For instance, Bänsch and Götz ${ }^{55}$ presented a rich numerical model that considers various physical mechanisms involved in the evaporation of levitating drops. Their model takes into account the geometry of the levitator and can predict the drop position and shape, the air flow due to acoustic streaming, as well as the evaporation rate, vapor transport, and temperature distribution.

Most studies involving the acoustic levitation of drops employ a single-axis levitator which consists of an ultrasonic emitter and a reflector opposite to it. A standing wave of high-pressure amplitude is generated between the emitter and the reflector, allowing the suspension of drops slightly below the pressure nodes. The distance between the emitter and the reflector has to be adjusted to achieve resonance and obtain high pressure amplitude. The acoustic levitation of drops using single-axis levitators has been extensively studied, but there are still multiple open questions regarding the stability of the levitated drops. For example, it was reported that only small drops can be stably levitated if the transducer-reflector distance is smaller than the resonant distance. ${ }^{47}$ In this situation, other drops [i.e., diameters larger than $3-4 \mathrm{~mm}(\approx 0.15 \lambda-0.20 \lambda)$ ] are atomized. These larger drops can only be levitated if the transducer-reflector distance is slightly larger than the resonant distance. ${ }^{47}$ In addition, when the levitator operates above the resonance distance, the levitated sample suffers an oscillation instability. ${ }^{56,57}$ In this type of instability, the sample oscillates vertically with constant amplitude, or its amplitude grows exponentially until the object hits the levitator wall. This type of instability seems to be the cause of the levitated dancing drops observed by Lin and Lin. ${ }^{5}$

Most of the models from previous studies ${ }^{43,44,46,50}$ use an incident field based on a plane standing wave. However, this field does not capture all the characteristics of the incident field generated inside an experimental levitator and cannot explain how the transducer-reflector distance or the insertion of the sample affects the levitation.

In this paper, we present a numerical model that combines the Finite Element Method (FEM) with the Young-Laplace equation to simulate the acoustic levitation of a liquid drop inside a single-axis acoustic levitator. The model simulates the acoustic pressure and velocity fields to predict the equilibrium position and shape of the levitating drop. Using this model, we investigate the acoustic levitation of water drops when the levitator is driven below and above resonance. We also conduct a series of experiments to obtain the static shape of the drop for different levitator parameters and we use a high-speed camera to observe the undesired oscillations and the disintegration of water drops.

\section{NUMERICAL MODEL}

In this section, a numerical model for simulating the shape of a drop inside a single-axis acoustic levitator is presented. The numerical model uses a finite element method to solve the linear wave equation and find the acoustic pressure and velocity amplitude fields in the propagation medium (i.e., air). These fields are used for calculating the acoustic radiation pressure exerted at each point of the drop surface, and then, the equilibrium shape of a droplet is determined by balancing the acoustic radiation pressure with the hydrostatic and capillary pressures using the Young-Laplace equation. To simplify the analysis, we assume an inviscid fluid and we neglect acoustic streaming ${ }^{37,58-60}$ and the generation of harmonics of the fundamental frequency. ${ }^{61,62}$

\section{A. Acoustic radiation pressure on the levitating drop}

The acoustic radiation pressure distribution over the drop surface is determined by performing an acoustic simulation in the FEM software COMSOL Multiphysics (COMSOL AB, Stockholm, Sweden). The drop is levitated inside a single-axis acoustic levitator which consists of a circular ultrasonic transducer separated by a distance $H$ from a plane reflector. The transducer radiating surface vibrates harmonically with frequency $f$ and velocity amplitude $u_{0}$. Due to the circular symmetry of the levitator, the simulations are carried out using an axisymmetric model [Fig. 1(a)], where the acoustic pressure $p$ and velocity $\mathbf{u}$ fields are described in terms of the spatial coordinates $r$ and $z$. A Perfectly Matched Layer (PML) is added to avoid wave reflections at the outer boundaries of the air domain. Due to the large acoustic impedance mismatch between the air-solid and air-liquid interfaces, only a very small fraction of the incident wave energy $(\approx 0.1 \%)$ penetrates into the drop. Therefore, we assume that the drop and the reflector are impenetrable to sound waves, which leads to the boundary condition $\mathbf{n} \cdot \nabla p=0$ at the airdrop and air-reflector interfaces, where $\mathbf{n}$ is the unit normal vector to the surface.

The model of Fig. 1(a) is used for calculating the acoustic pressure $p$ and the velocity amplitude $\mathbf{u}$ distributions in the air domain. Although $p$ and $\mathbf{u}$ are time-harmonic fields, an object can experience a nonzero radiation pressure due to nonlinear effects. ${ }^{21,63}$ Therefore, after obtaining the $p$ and $\mathbf{u}$ fields, the timeaveraged radiation pressure distribution over the drop surface can be calculated by ${ }^{18,21}$

$$
p_{\text {rad }}=\frac{1}{2 \rho_{0} c_{0}^{2}}\left\langle p^{2}\right\rangle-\frac{\rho_{0}}{2}\langle\mathbf{u} \cdot \mathbf{u}\rangle
$$

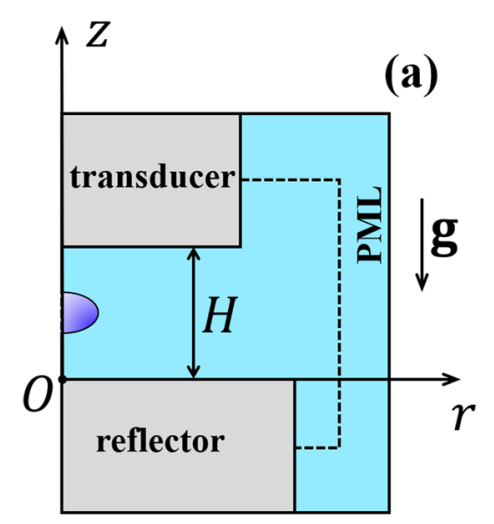

(b)

FIG. 1. Numerical model. (a) Axisymmetric acoustic model used to find the acoustic pressure and particle velocity distributions in the air medium. (b) Drop geometry in spherical polar coordinates. 
where $\rho_{0}$ is the unperturbed air density, $c_{0}$ is the speed of sound in air, and the symbol \langle\rangle denotes the time-averaged over one cycle. The radiation pressure given by Eq. (1) depends on two terms. The first term implies that zones of high acoustic pressure exert a positive pressure over the object surface whereas the second term represents the Bernoulli effect, in which the oscillatory motion of the air particles causes a negative pressure on the object. The acoustic radiation force $\mathbf{F}_{\text {rad }}$ on the drop is then calculated by integrating the radiation pressure over the drop surface $S_{0}$,

$$
\mathbf{F}_{\text {rad }}=-\int_{S_{0}} p_{\text {rad }} \mathbf{n} d S .
$$

In Eq. (2), $\mathbf{n}$ is the normal unit vector pointing outward from the surface $S_{0}$. With a procedure similar to that adopted by Hong et al., ${ }^{64}$ Eq. (2) is also used to obtain the acoustic radiation force acting on the reflector as a function of $H$.

In the FEM simulations, the air domain was simulated using a nonuniform mesh with a size of $50 \mu \mathrm{m}(\lambda / 272)$ at the drop surface and a maximum mesh size of $1 \mathrm{~mm}(\lambda / 13.6)$ at the edge of the air domain, which results in a convergence error of less than $0.5 \%$ for the acoustic pressure and less than $2 \%$ for the acoustic radiation force acting on the drop.

\section{B. Determination of the position and static shape of the levitating drop}

In this section, the numerical algorithm to obtain the equilibrium position and the static shape of the drop is described. In the presence of a gravitational field $\mathbf{g}$, the drop is suspended slightly below the pressure node of the standing wave. For small displacements compared to the pressure node, the radiation force can be approximated as the restoring force of a spring, ${ }^{18,65}$ with a stiffness proportional to the pressure amplitude squared. The equilibrium is reached when the gravity force acting on the drop is counterbalanced by the acoustic radiation force.

A levitating drop is flattened due to the action of the acoustic radiation force and its equilibrium shape can be obtained by solving the Young-Laplace equation,

$$
p_{\text {ext }}-p_{\text {in }}=-\gamma \nabla \cdot \mathbf{n},
$$

where $p_{\text {ext }}$ and $p_{\text {in }}$ are the external and internal pressures, $\gamma$ is the surface tension, and $\nabla \cdot \mathbf{n}$ is the divergence of the drop surface normal. By taking into account the radiation pressure and the hydrostatic pressure inside the drop, Eq. (3) can be written as ${ }^{46,55}$

$$
p_{\text {rad }}+\rho_{l} g z+\gamma \nabla \cdot \mathbf{n}=C,
$$

where $\rho_{l}$ is the density of the liquid, $g$ is the gravity acceleration, $z$ is the spatial coordinate, and $C$ is a constant that includes the ambient pressure.

The vertical drop position is represented by $z_{d}$ and the drop shape is described in spherical polar coordinates [Fig. 1(b)] by the function $r(\theta)$. In Fig. 1(b), the surface normal vector is written as $\mathbf{n}=n_{\boldsymbol{r}} \hat{\boldsymbol{r}}+n_{\boldsymbol{\theta}} \hat{\boldsymbol{\theta}}$.

Given a drop volume, the working parameters of the levitator, and an initial estimation of the position and shape of the drop, the objective is to find $z_{d}$ and $r(\theta)$ that satisfy simultaneously Eq. (4) and $\mathbf{F}_{\text {rad }}=-m \boldsymbol{g}$. The algorithm was implemented in MATLAB. At each iteration, the FEM model of Fig. 1(a) is executed through the
LiveLink feature of the software COMSOL Multiphysics. Each iteration $i$ starts with the determination of the equilibrium vertical position $z_{d}$ of the drop. This is done by using the Nelder-Mead method to find $z_{d}^{i+1}$ satisfying $\mathbf{F}_{\text {rad }}=-m \boldsymbol{g}$ for a given drop shape $r^{i}(\theta)$. Then, the FEM model calculates the radiation pressure $p_{\text {rad }}(\theta)$ over the surface of the drop and $r^{i+1}(\theta)$ at the step $i+1$ is calculated by

$$
r^{i+1}(\theta)=r^{i}(\theta)+\alpha \frac{\left(h-p_{r a d}-\gamma \nabla \cdot \mathbf{n}-\rho_{l} g z\right)}{n_{r}},
$$

where $\alpha$ is a constant, with a typical value between $10^{-9}$ and $10^{-7}$ $\mathrm{m} / \mathrm{Pa}$, and $h$ is given by ${ }^{55}$

$$
h=\frac{\int_{S_{0}}\left(p_{\text {rad }}+\gamma \nabla \cdot \mathbf{n}+\rho_{l} g z\right) d S}{\int_{S_{0}} d S} .
$$

This step was based on the numerical method presented by Bänsch and Götz. ${ }^{55}$ Because our algorithm calculates the radiation pressure at each iteration, it is less efficient than that of Bänsch and Götz. However, this modification allows us to visualize the acoustic pressure distribution at each iteration, providing a physical insight into how the drop shape affects the acoustic pressure amplitude. If needed, the algorithm can be executed faster, by reiterating Eqs. (5) and (6) multiple times without recalculating $p_{\text {rad }}(\theta)$ at each reiteration.

At the end of each iteration, the volume of the resulting drop shape is calculated and $r^{i+1}(\theta)$ is multiplied by $\left(V_{\text {initial }} / V_{\text {current }}\right)^{1 / 3}$, where $V_{\text {initial }}$ is the initial drop volume and $V_{\text {current }}$ is the current

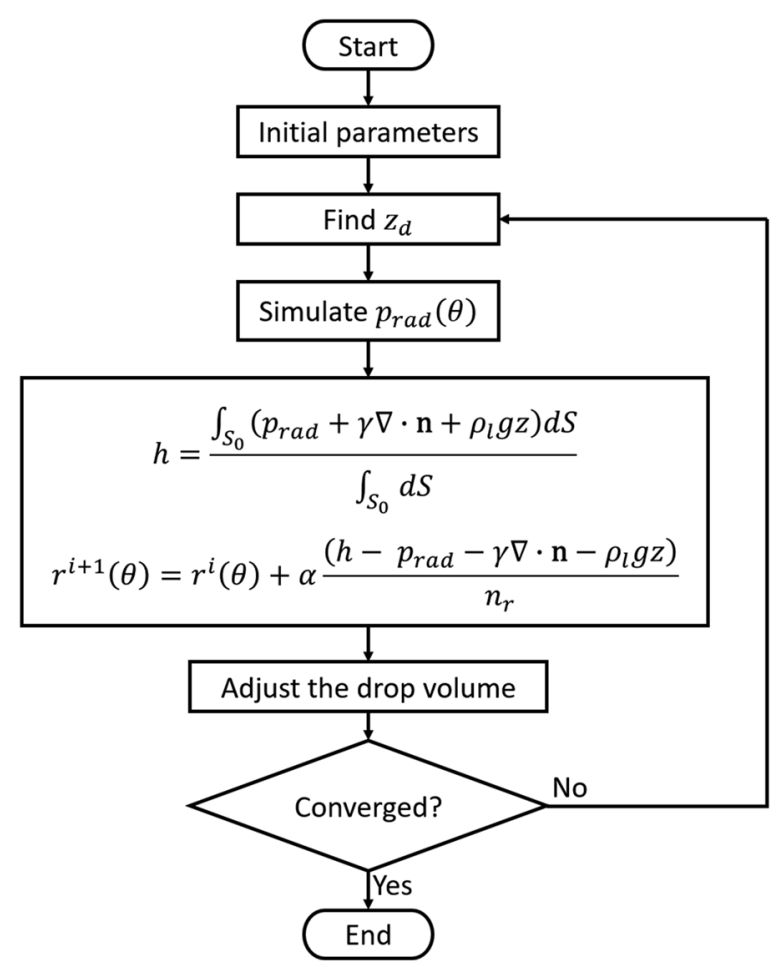

FIG. 2. Flowchart of the algorithm used to obtain the shape and equilibrium position of the drop. 
TABLE I. Simulation parameters.

\begin{tabular}{llc}
\hline \hline Symbol & \multicolumn{1}{c}{ Description } & Value \\
\hline$f$ & Frequency & $25.25 \mathrm{kHz}$ \\
$c_{0}$ & Speed of sound in air & $343 \mathrm{~m} / \mathrm{s}$ \\
$\rho_{0}$ & Unperturbed air density & $1.2 \mathrm{~kg} / \mathrm{m}^{3}$ \\
$\rho_{l}$ & Density of water & $1000 \mathrm{~kg} / \mathrm{m}^{3}$ \\
$\gamma$ & Surface tension of water & $72 \mathrm{mN} / \mathrm{m}^{2}$ \\
$g$ & Gravitational acceleration & $9.8 \mathrm{~m} / \mathrm{s}^{2}$ \\
$R_{t}$ & Transducer radius & $10 \mathrm{~mm}$ \\
$R_{r}$ & Reflector radius & $19 \mathrm{~mm}$ \\
\hline \hline
\end{tabular}

drop volume. This procedure preserves the drop shape and maintains the volume constant ${ }^{46}$ at the same time. The flowchart of the algorithm is presented in Fig. 2, and the parameters used in all simulations are presented in Table I.

\section{EXPERIMENTAL SETUP}

Water drops were levitated in a single-axis acoustic levitator operating at a frequency of $25.25 \mathrm{kHz}$ under the first acoustic mode $(H \approx \lambda / 2)$, where $\lambda=13.58 \mathrm{~mm}$ is the acoustic wavelength. The levitator consists of a Langevin-type transducer with a circular radiating surface of $20 \mathrm{~mm}$ in diameter on top of a 38-mm-diameter reflector. The transducer signal is generated by a programmable function generator (Keysight, 33512B) fed to a high-power amplifier (Amplifier Research Corp., 700A1). The transducer was mounted on a motorized linear stage, allowing to adjust the distance between the transducer and the reflector with a resolution of $0.1 \mu \mathrm{m}$. The acoustic radiation force on the reflector is measured using a scale (Shimadzu, UX420H) with $1 \mathrm{mg}$ resolution. A MATLAB script was implemented to control the transducer voltage amplitude and the motorized stage and to acquire the radiation forces measured by the scale. The images and videos of the levitating drops were captured by a high-speed camera (Photron FASTCAM Mini UX50). The experimental setup is illustrated in Fig. 3, and more details can be found in previous work on levitating solid particles.

To calculate the pressure amplitude $p_{0}$ at the center of the reflector surface $(r=0, z=0)$, we measure the radiation force exerted

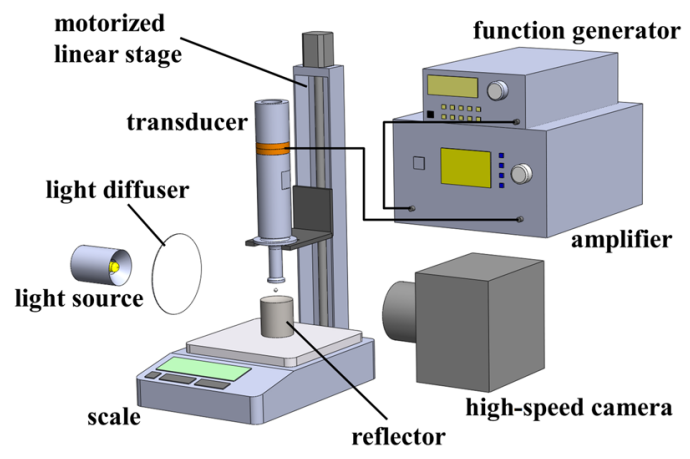

FIG. 3. Schematic of the experimental setup on the reflector with the scale and then we apply the following relationship:

$$
p_{0}=\beta \sqrt{F_{\text {reflector }}},
$$

where $F_{\text {reflector }}$ is the radiation force on the reflector and $\beta=77850 \mathrm{~Pa}$ $\mathrm{N}^{-1 / 2}$ is the constant that relates the pressure amplitude with the force on the reflector. This constant was obtained using the numerical model of Fig. 1(a), which was used to calculate the radiation force on the reflector and the acoustic pressure distribution in the air medium.

\section{RESULTS AND DISCUSSION}

In all the experiments, the levitator is excited at a frequency of $25.25 \mathrm{kHz}$. The levitator is tuned by adjusting the distance $H$ between the transducer and the reflector. The resonance is found by locating the peak of acoustic radiation force on the reflector as a function of $H$ without the drop inserted. The force on the reflector was measured for $H$ varying from 6.5 to $8.5 \mathrm{~mm}$, with the transducer face oscillating with a velocity amplitude of $1 \mathrm{~m} / \mathrm{s}$. According to this measurement (Fig. 4-dashed red line), the resonance peak for the empty cavity (i.e., nothing inside the levitator) occurs at $H=7.50 \mathrm{~mm}(0.55 \lambda)$. This value is in good agreement with the resonance obtained numerically (Fig. 4-solid black line), which corresponds to $H=7.44 \mathrm{~mm}$.

We investigate the acoustic levitation of a drop when the levitator operates below (point A of Fig. 4) and above (point B) the resonance. Point $A$ is located $0.2 \mathrm{~mm}$ before the resonance peak and point $\mathrm{B}$ occurs $0.2 \mathrm{~mm}$ after the resonance.

\section{A. Resonance shift}

The introduction of a drop inside the levitator causes a resonance shift, which depends on the position of the object, its volume, and its shape. ${ }^{66}$ For small sound intensities, the levitating drop is almost spherical, and it becomes more flattened as the sound intensity increases. For moderate sound intensities, the drop presents a

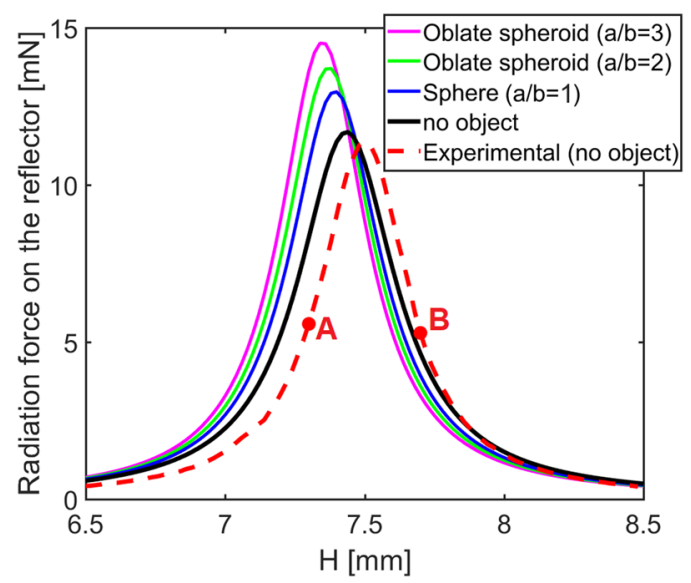

FIG. 4. Acoustic radiation force on the reflector as a function of $H$ with no object in the levitator and with an oblate spheroid of constant volume and different aspect ratios. The spheroid has an equivalent spherical radius of $1 \mathrm{~mm}$. 
shape close to an oblate spheroid. In order to investigate how the shape of a drop of constant volume affects the resonance of the levitator, we simulate the acoustic radiation force on the reflector as a function of $H$ for different shapes of the levitating drop. We assume that an oblate spheroid drop of constant volume (equivalent spherical radius of $1 \mathrm{~mm}$ ), equatorial radius $a$, and polar radius $b$ is located at a height $z=H / 2$. In all the simulations, the transducer vibrates with a velocity amplitude of $1 \mathrm{~m} / \mathrm{s}$.

The radiation force on the reflector for different aspect ratios $(a / b)$ of the spheroid is shown in Fig. 4. For an empty levitator, the simulated resonance distance is $H=7.44 \mathrm{~mm}$ (Fig. 4-peak of the black curve). The introduction of an oblate spheroid of different aspect ratios causes a decrease in the resonance distance. As shown in Fig. 4, the resonance distances are $H=7.40 \mathrm{~mm}$ (sphere or spheroid with $a / b=1$ ), $7.38 \mathrm{~mm}$ (spheroid with $a / b=2$ ), and $7.34 \mathrm{~mm}$ (spheroid with $a / b=3$ ). This result shows that even if the drop volume remains constant, the resonance distance decreases as the drop becomes more flattened.

As we will see in Sec. IV B, the resonance shift is of vital importance for explaining why levitating drops are disintegrated when they exceed a certain aspect ratio if $H$ is smaller than the resonance distance. The resonance shift also helps us to explain why drops can take the flattened shape of a pancake when $H$ is greater than the resonance distance.

\section{B. Static shape and disintegration of the levitating drop}

The static shape of a levitating water drop was determined numerically for different conditions of the levitator. The result of a typical simulation is presented in Fig. 5. This simulation was carried out by considering a water drop of $1 \mathrm{~mm}$ spherical radius suspended at the pressure node when the transducer surface vibrates with a velocity amplitude of $u_{0}=1 \mathrm{~m} / \mathrm{s}$, and it is located at a distance of $7.64 \mathrm{~mm}$ from the reflector (i.e., $0.2 \mathrm{~mm}$ above the resonance distance-Fig. 4 point A). The evolution of the drop shape after each iteration of the algorithm can be seen in an online video [Fig. 5
(Multimedia View)]. The algorithm starts by assuming a spherical drop of $1 \mathrm{~mm}$ radius, and it is executed until an equilibrium shape for the drop is obtained. The online video [Fig. 5 (Multimedia View)] also shows the evolution of the pressure amplitude at the position $(r=0, z=0)$. Because the levitator is operating above the resonance, the resonance shift causes a decrease in the pressure amplitude when the drop changes its shape from a sphere to an oblate spheroid.

The acoustic pressure and velocity fields for the equilibrium shape of the drop are presented in Figs. 5(a) and 5(b), respectively. These figures show that the pressure amplitude is maximum at the poles, and the particle velocity is maximum at the equator of the drop. The radiation pressure $p_{\text {rad }}$ over the drop surface calculated using Eq. (1) is shown in Fig. 5(c). This figure also shows the contribution of the first $\left(\left\langle p^{2}\right\rangle / 2 \rho_{0} c_{0}^{2}\right)$ and second $\left(-\rho_{0}\langle\mathbf{u} \cdot \mathbf{u}\rangle / 2\right)$ terms on the total radiation pressure. The vertical force, responsible for the levitation, is mainly dominated by the first term, whereas the second term is the main responsible of the lateral trapping force. Additionally, as it can be seen in Figs. 5(c) and 5(d), the second term produces a suction force at the equator, causing a flattening effect on the drop.

In Fig. 6, we show the comparison between the equilibrium shapes of water drops obtained numerically and experimentally. These results were obtained for the levitator operating with a transducer-reflector distance of $0.2 \mathrm{~mm}$ below the resonance distance (point A). Because of the small mismatch between numerical and experimental curves in Fig. 4, the experiments were carried out with a transducer-reflector distance of $H=7.30 \mathrm{~mm}$, whereas the simulations were performed with a separation distance of $H=7.24 \mathrm{~mm}$. To obtain the experimental results of Fig. 6, the radiation force on the reflector was measured by the scale, and pictures of the levitating drop were taken with the high-speed camera for different transducer voltage amplitudes. Then, the force values were converted to acoustic pressure amplitude using Eq. (7). As shown in Fig. 6(c), there is a good agreement between numerical and experimental results, in terms of both the vertical equilibrium position and the shape of the levitating drops. The simulation also shows that an equilibrium shape for the drop can only be obtained if the
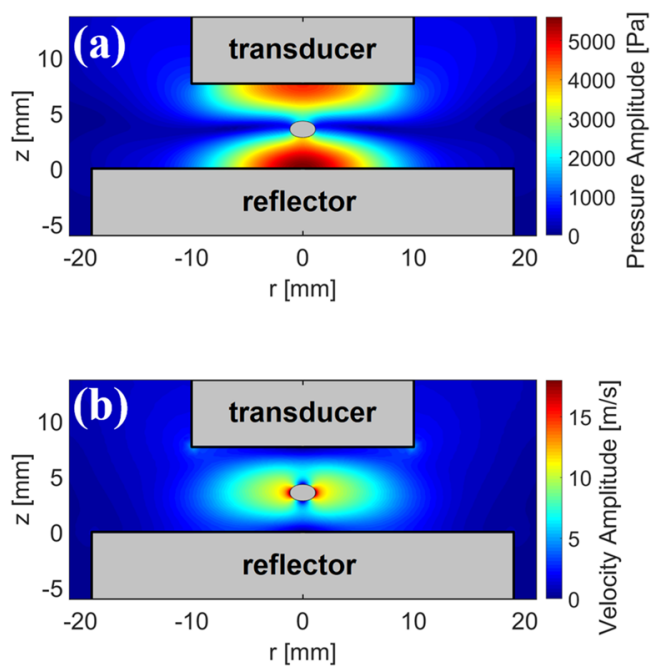

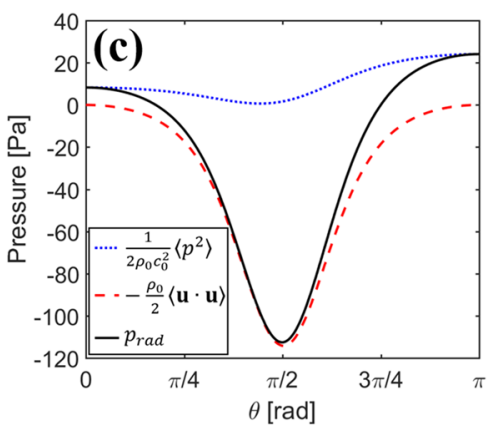

(d)

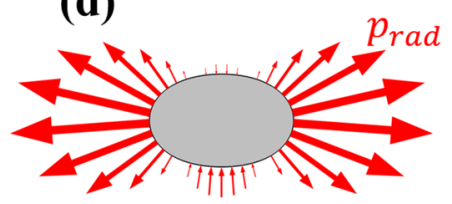

FIG. 5. Simulated results for a levitating water drop of $1 \mathrm{~mm}$ spherical radius. The simulation was carried out with $H=7.64 \mathrm{~mm}$ and $u_{0}=1 \mathrm{~m} / \mathrm{s}$. (a) Acoustic pressure field. (b) Velocity amplitude distribution. (c) Distribution of the radiation pressure over the drop surface as a function of the polar angle $\theta$. (d) Illustration of the acoustic radiation pressure acting on the drop surface. A video showing the evolution of the drop shape for each iteration step is available online. Multimedia view: https://doi.org/10.1063/1.5121728.1 

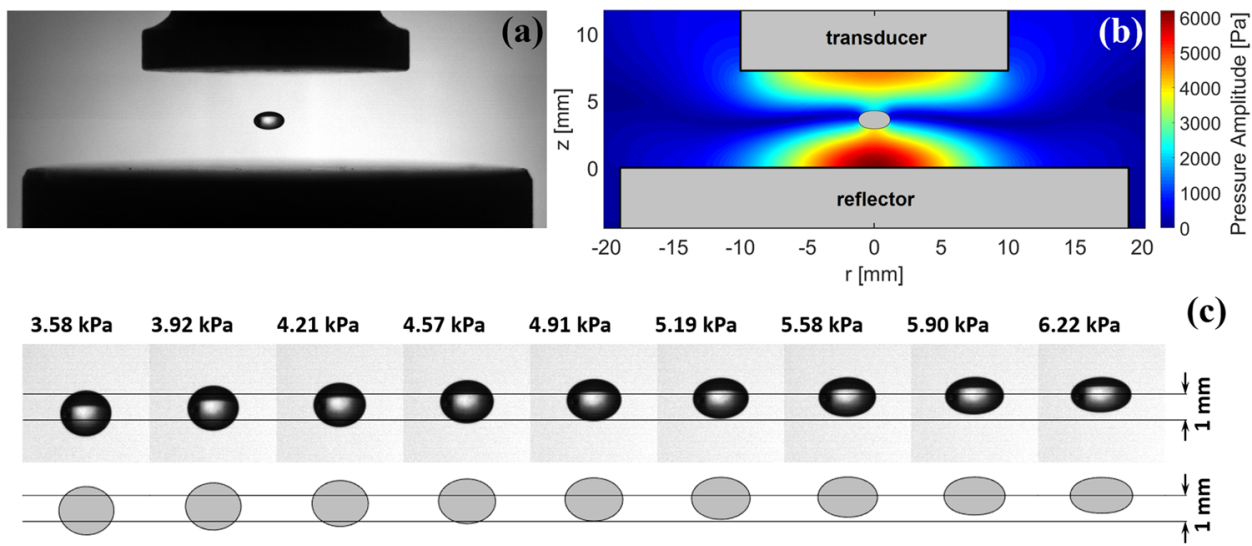

$5.19 \mathrm{kPa}$
$5.58 \mathrm{kPa}$
$5.90 \mathrm{kPa}$
$6.22 \mathrm{kPa}$

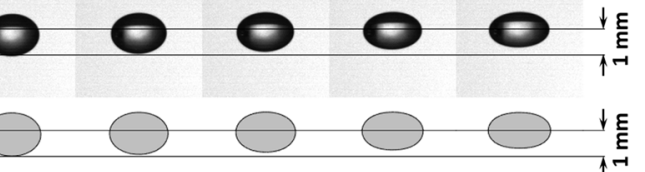

FIG. 6. Comparison between equilibrium drop shapes obtained numerically and experimentally for a water drop of $1 \mathrm{~mm}$ spherical radius, $H=7.24 \mathrm{~mm}$ and different acoustic pressure amplitude (pressure control by the excitation voltage of the transducer). (a) Experimental (pressure amplitude of $6.22 \mathrm{kPa}$ ). (b) Numerical (pressure amplitude of $6.22 \mathrm{kPa}$ ). (c) Drop shape as a function of the pressure amplitude obtained numerically and experimentally. An online video shows that the drop disintegrates when the transducer vibrates with a velocity amplitude of $0.87 \mathrm{~m} / \mathrm{s}$. Multimedia view: https://doi.org/10.1063/1.5121728.2 transducer vibrates with a velocity amplitude smaller than $0.87 \mathrm{~m} / \mathrm{s}$. In contrast with the case where the levitator operates above the resonance, here the flattening of the drop causes an increase in the pressure amplitude due to the resonance shift effect. When the transducer velocity amplitude is increased from $0.86 \mathrm{~m} / \mathrm{s}$ to $0.87 \mathrm{~m} / \mathrm{s}$, there is a rapid increase in the acoustic pressure amplitude, causing the rapid expansion of the drop equatorial radius [see online video Fig. 6 (Multimedia view)].

For a transducer-reflector distance below the resonance distance, the levitating drop disintegrates when the aspect ratio exceeds a certain value. To observe the drop disintegration, the transducer voltage amplitude was raised slowly, causing a slow increase in the drop aspect ratio. When the pressure amplitude reaches a threshold value, there is an abrupt expansion of the equatorial radius, followed by the drop disintegration. For a water drop of $1 \mathrm{~mm}$ spherical radius and $H=7.24 \mathrm{~mm}$, the disintegration process starts when the aspect ratio reaches $a / b=2.1$. The drop disintegration can be seen in Fig. 7, which also shows the equatorial radius as a function of time; it takes approximately $5 \mathrm{~ms}$ for the equatorial radius to change from $1.3 \mathrm{~mm}$ to $3.0 \mathrm{~mm}$, where the disintegration occurs. This result is in qualitative agreement with the experimental results obtained by Anilkumar et $a l^{49}$ and with the numerical results obtained by Shi et al. ${ }^{5}$

The resonance shift plays an important role in the drop disintegration shown in Fig. 7. Without resonance shift, the acoustic pressure amplitude would be proportional to the transducer velocity amplitude. However, as the levitator is operating below the resonance distance, the flattening of the drop causes an increase in the acoustic pressure amplitude. Consequently, when the aspect ratio exceeds a threshold value, a small increase in the pressure amplitude causes a further flattening of the drop, which causes a new increase in the pressure amplitude and so on. This process contributes to the rapid expansion of the equatorial radius, as shown in Fig. 7 (Multimedia view).

The numerical simulation shown in Fig. 6 can be used to explain why the drop disintegrates when the drop aspect ratio reaches a certain value. According to the simulations presented in Fig. 6, a stable levitation can only be achieved if the transducer velocity amplitude is lower than $0.86 \mathrm{~m} / \mathrm{s}$. For this velocity amplitude and the levitator operating below the resonance $(H=7.24 \mathrm{~mm})$, a water drop of $1 \mathrm{~mm}$ spherical radius takes the shape of an oblate
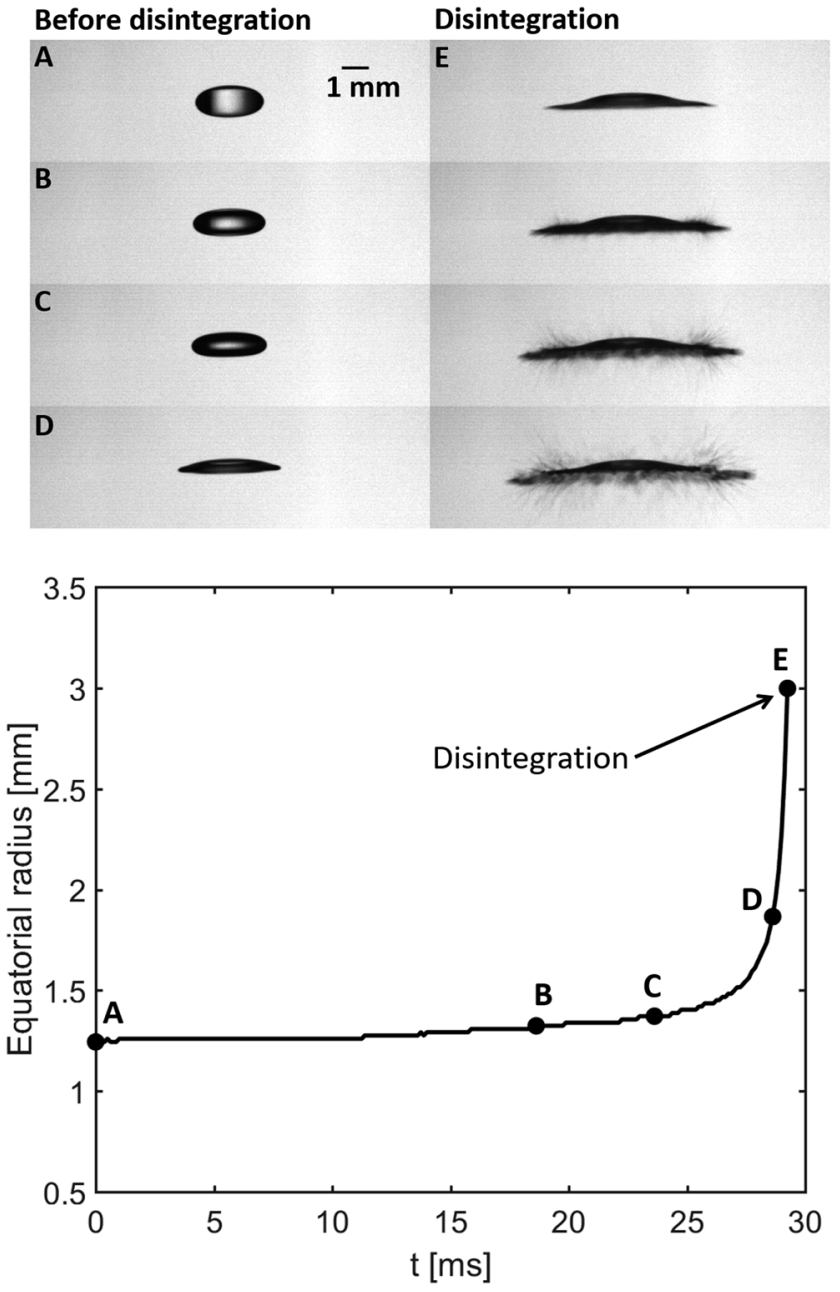

FIG. 7. Disintegration of a water drop of $1 \mathrm{~mm}$ spherical radius when the levitator is driven below the resonance $(H=7.24 \mathrm{~mm})$. The bottom figure shows the equatorial radius as a function of time. A video of the drop disintegration is available online. Multimedia view: https://doi.org/10.1063/1.5121728.3 
spheroid, with an aspect ratio of $a / b=2.4$, which is slightly larger than the aspect ratio of 2.1 found experimentally. In this condition, the pressure amplitude corresponds to $7051 \mathrm{~Pa}$. When the velocity amplitude is increased from $0.86 \mathrm{~m} / \mathrm{s}$ to $0.87 \mathrm{~m} / \mathrm{s}$, the pressure amplitude rapidly increases, causing the drop disintegration. This increase in the pressure amplitude is caused by the resonance shift effect and it can be observed in an online video [Fig. 6 (Multimedia view)].

In contrast with the former case, an acoustic levitator operating above the resonance distance allows a substantial flattening of the drop, up to the point in which it takes the shape of a pancake. This behavior can be observed in Fig. 8, which shows the acoustic pressure amplitude as a function of the equatorial radius for a water drop of $1.2 \mathrm{~mm}$ spherical radius. To obtain this figure, a flattened water drop of $2 \mathrm{~mm}$ equatorial radius was levitated (Fig. 8-frame A). Then, the transducer velocity amplitude was increased, causing the further flattening of the drop (frames A-E in Fig. 8). However, instead of the linear increase in the pressure amplitude with the transducer velocity amplitude, the resonance shift due to the flattening of the drop causes a decrease in the pressure amplitude. This effect was also observed by Anilkumar et al., ${ }^{49}$ but in our experiments, this effect only occurs if the levitator operates above the resonance distance.

A further increase in the transducer velocity amplitude causes the atomization of the drop (Fig. 9). In our experiments, the atomization process can be divided in the following stages: (1) flattening, (2) capillary wave formation, and (3) atomization. These stages are illustrated in Fig. 9(d). First, the drop changes its shape from an oblate spheroid to a pancake shape, with its rim having a greater thickness in comparison to its center. At some point, a capillary wave grows on the central surface of the drop [Fig. 9(b)]. When the amplitude of the capillary wave reaches a critical value, atomization occurs on the central part [Figs. 9(a) and 9(c)]. The atomization of a drastically flattened drop can also be observed in the online videos [Figs. 9(a) and 9(c) (Multimedia view)]. A mechanism for the growing of capillary waves on the surface of the drop and its atomization was suggested by Danilov and Mironov. ${ }^{67}$ According to their
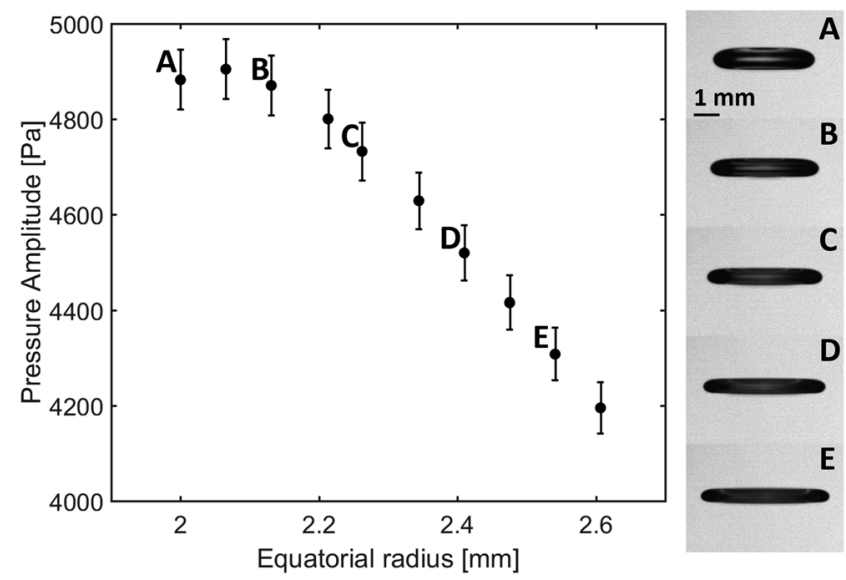

FIG. 8. Acoustic pressure amplitude as a function of the equatorial radius for a water drop of $1.2 \mathrm{~mm}$ spherical radius when the levitator is driven above the resonance $(H=7.64 \mathrm{~mm})$.
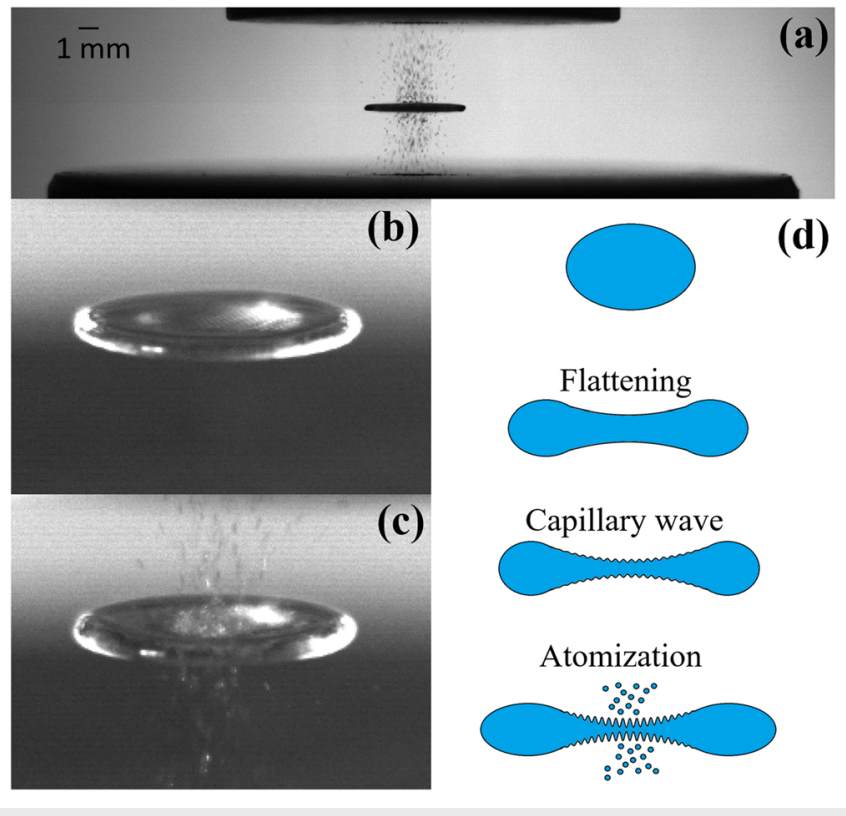

FIG. 9. Atomization of a drastically flattened drop. (a) Atomization of a water drop-side view. (b) Flattened drop with capillary waves on its surface. (c) Atomization of a water drop. (d) Stages of the atomization process. Videos showing the atomization of the drop are available online. Multimedia views: (a) https://doi.org/10.1063/1.5121728.4; (c) https://doi.org/10.1063/1.5121728.5

theoretical analysis, when the drop becomes sufficiently thin, the central drop membrane vibrates vertically at the same frequency of as the sound wave. Then, capillary standing waves grow along the air-liquid interface due to the parametric instability. The atomization of the central membrane starts when the transducer velocity amplitude is increased above a critical value and small droplets are ejected from the crests of the capillary waves. Although our experimental results are in qualitative agreement with the physical mechanisms suggested by Danilov and Mironov, ${ }^{67}$ we acknowledge that future quantitative analysis would be desirable for investigating the atomization of levitating drops.

The stages involved in the breakup of a levitating drop do not necessarily follow the stages shown in Fig. 9(d). Depending on the conditions of the experiment and on the levitator geometry, the drop breakup can have other stages. For example, in the experiments of Lee et al., ${ }^{43}$ Pathak and Basu, ${ }^{68}$ Zang et al., ${ }^{69}$ and Di et al., ${ }^{70}$ the drop was also subjected to buckling, which is characterized by a nonuniform radiation pressure distribution below and above the drop surface, that makes a flattened drop buckle downward or upward. In the experiments reported by Zang et al., ${ }^{69}$ the abrupt buckling of a flattened levitating drop was a fundamental stage for transforming a drop into a bubble.

It has also been observed experimentally that if the levitator is driven below the resonance, only small drops can be suspended. To understand why, we simulated the levitation of water drops of different sizes (spherical radius of $1,1.5$, and $2 \mathrm{~mm}$ ) for the levitator operating below the resonance $(H=7.14 \mathrm{~mm})$. As shown in Fig. 10, a water drop of $1 \mathrm{~mm}$ spherical radius can only be levitated if the 


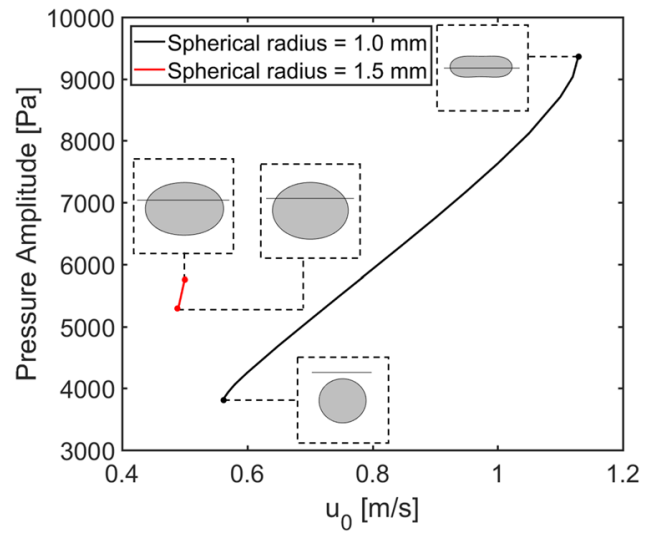

FIG. 10. Simulated acoustic pressure amplitude and drop shape as a function of the transducer velocity amplitude for the levitator operating below the resonance $(H=7.14 \mathrm{~mm})$ when levitating a drop with spherical radius $1 \mathrm{~mm}$ (black line) or $1.5 \mathrm{~mm}$ (red line).

transducer velocity amplitude is set between $0.56 \mathrm{~m} / \mathrm{s}$ and $1.13 \mathrm{~m} / \mathrm{s}$. If the velocity amplitude is lower than $0.56 \mathrm{~m} / \mathrm{s}$, the acoustic radiation force acting on the drop is not strong enough to counteract gravity, and if the velocity amplitude exceeds $1.13 \mathrm{~m} / \mathrm{s}$, the drop disintegrates.

According to the simulation results, a water drop of $1.5 \mathrm{~mm}$ spherical radius can only be levitated in a very small range of transducer velocity amplitudes. As shown in Fig. 10, the minimum velocity amplitude is $0.49 \mathrm{~m} / \mathrm{s}$ and the maximum amplitude is $0.50 \mathrm{~m} / \mathrm{s}$. For a drop of $2 \mathrm{~mm}$ spherical radius, we could not find any acceptable values of velocity amplitudes, which means that the levitator is unable to levitate water drops of this size when it is operating below resonance.

Although the simulation predicted that water drops of $1.5 \mathrm{~mm}$ equivalent spherical radius could be levitated below the resonance, in the experiments, we could only levitate water drops with a maximum spherical radius of $1.2 \mathrm{~mm}$. When trying to levitate water drops larger than $1.2 \mathrm{~mm}$ below the resonance, either the radiation force was not strong enough to hold the drop, or the acoustic pressure amplitude was too high and caused the drop disintegration. In the experiments, larger drops could only be levitated for the levitator operating above the resonance distance.

\section{Oscillation instability of levitating drops}

When the levitator operates above the resonance distance, the levitating drops are subjected to oscillation instability. ${ }^{56,57}$ For a rigid object, the oscillation instability can make the object oscillate vertically. As described by Rudnick and Barmatz, ${ }^{56}$ the oscillation instability is caused by two coupled factors: (1) The resonance frequency of the levitator changes with the object position, and (2) there is a time delay in the response of the pressure amplitude due to the vertical motion of the levitated object. These factors together result in a velocity-dependent term in the object's equation of motion. If the levitator operates above the resonance distance, the velocitydependent term takes the form of a negative damping, causing the exponential increase in the oscillation amplitude until the oscillation saturates or the object is ejected out of the levitator. In addition to the vertical oscillations on the position, the oscillation instability can also lead to shape oscillations of a liquid drop.

The oscillation instability of a water drop of $1 \mathrm{~mm}$ spherical radius was observed in the levitator operating above the resonance $(H=7.64 \mathrm{~mm})$. In the experiment, a stable levitation (i.e., no oscillation instability) was achieved for a pressure amplitude below $5150 \mathrm{~Pa}$. When the transducer velocity amplitude is increased, the pressure amplitude rises, causing shape oscillations of the levitating drop. The shape oscillation of a water drop for a pressure amplitude of $5815 \mathrm{~Pa}$ drop can be observed in Fig. 11 and in an online video
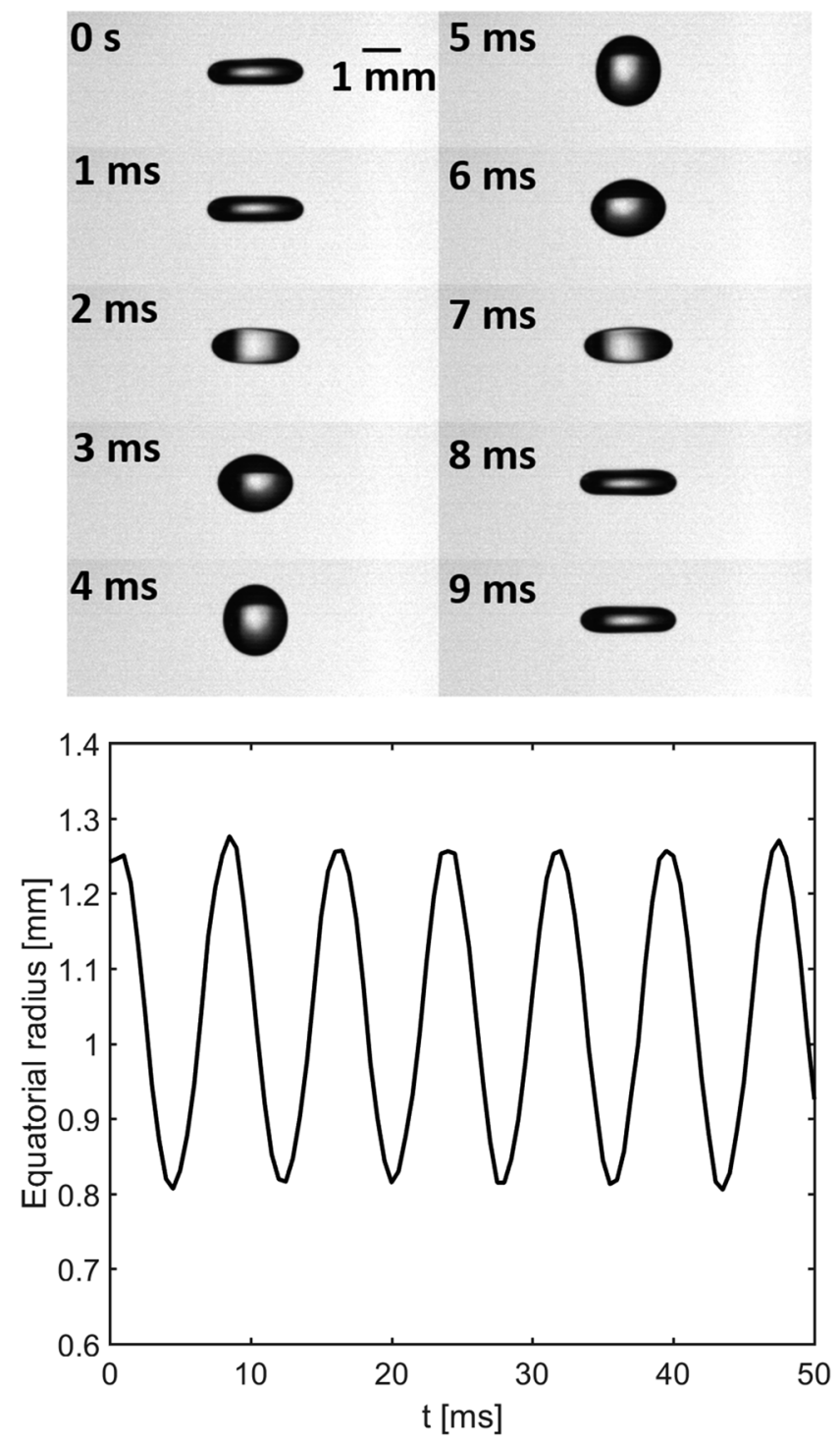

FIG. 11. Shape oscillation of a water drop of $1 \mathrm{~mm}$ spherical radius. The distance between the emitter and the reflector is $H=7.64 \mathrm{~mm}$. A video showing the shape oscillation of the levitated drop is available online. Multimedia view: https://doi.org/10.1063/1.5121728.6 
[Fig. 11 (Multimedia view)]. Figure 11 also shows the evolution of the equatorial radius over time. The resonance frequencies of the shape oscillations of a spherical drop can be calculated by ${ }^{7}$

$$
f_{l}=\frac{1}{2 \pi} \sqrt{\frac{\gamma}{\rho_{l} R^{3}} l(l-1)(l+2)},
$$

where $R$ is the drop spherical radius and $l$ is the resonance mode. In Fig. 12 , the drop oscillates under its first mode $(l=2)$ at a frequency of $128 \mathrm{~Hz}$. This value is in a reasonable agreement with the theoretical value obtained with Eq. (8), which corresponds to $120.8 \mathrm{~Hz}$. It is well known that shape oscillations can be induced by exciting the transducer with an amplitude modulated signal. For instance, amplitude modulated signals have been used to determine the surface tension ${ }^{35}$ of levitating drops and to excite different vibration modes of a drop. ${ }^{53}$ But here, the levitator is driven with a pure sinusoidal signal and the shape oscillations are caused by the oscillation instability.

An additional increase in the transducer velocity amplitude caused a transition from shape oscillation to vertical oscillations of the drop (Fig. 12). The vertical oscillation of Fig. 12 was observed when the pressure amplitude was $6170 \mathrm{~Pa}$. As shown in Fig. 12 and in an online video [Fig. 12 (Multimedia view)], the drop oscillates vertically at a frequency of $29.5 \mathrm{~Hz}$ with an amplitude of $0.35 \mathrm{~mm}$. Similarly to previous work on the oscillation of solid particles, ${ }^{5}$ the oscillation instability only occurs if the levitator is driven above the resonance distance. In Fig. 12, the drop oscillates with constant amplitude and frequency, but depending on the transducer
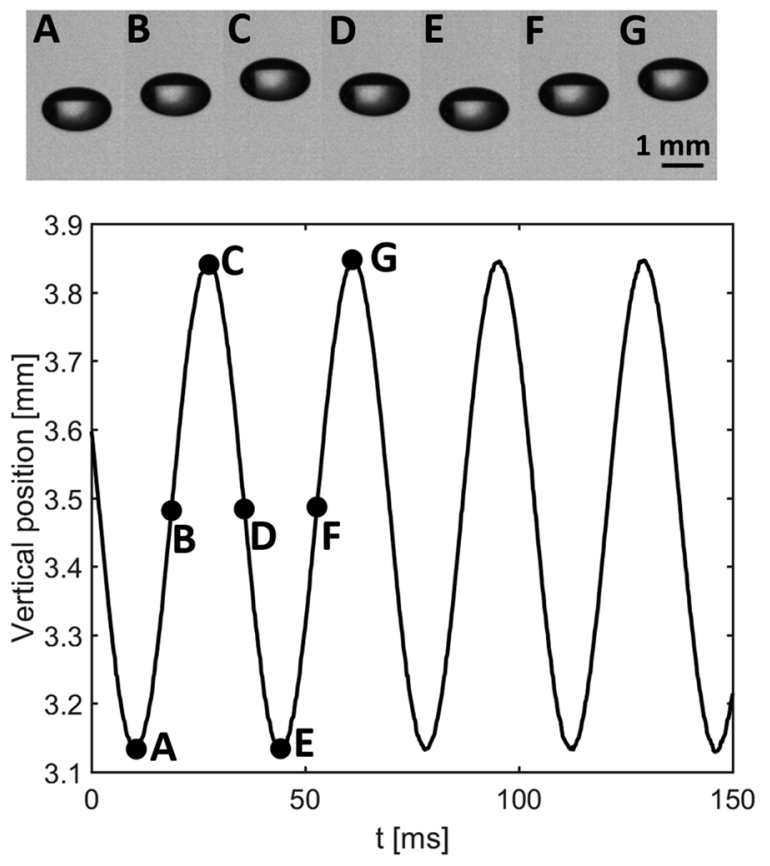

FIG. 12. Vertical position of a water drop of $1 \mathrm{~mm}$ equivalent spherical radius over time. The levitator is driven above the resonance $(H=7.64 \mathrm{~mm})$. A video showing the drop vertical oscillation is available online. Multimedia view: https://doi.org/10.1063/1.5121728.7 velocity amplitude and on the distance between the transducer and the reflector, the oscillation grows exponentially until the drop falls on the reflector.

Although the shape and vertical oscillations of the drop of Figs. 11 and 12 were observed separately, in general the vertical oscillation is coupled with the shape oscillation. Figure 13 (Multimedia view) shows the vertical and shape oscillations occurring simultaneously. This result was obtained when the pressure amplitude was increased to $6530 \mathrm{~Pa}$. The drop vertical oscillation has a frequency of $41.5 \mathrm{~Hz}$ and an amplitude of $0.7 \mathrm{~mm}$. This type of oscillation was previously observed by Lin and $\mathrm{Lin}^{54}$ and Geng et al. ${ }^{72}$ Similar to the observations of Geng et al. ${ }^{72}$ the drop becomes flattened when it reaches the minimum and maximum heights, but in their study the coupled oscillation did not started spontaneously, they used a needle to induce the vertical oscillation of the drop.

It was also observed that the coupled oscillation instability of Fig. 13 can be ceased by increasing the transducer velocity amplitude further, which causes the flattening of the drop into a pancake shape. This can be observed in the results of Fig. 8, which shows the stable levitation of a substantially flattened drop. In the experiment of Fig. 8, oscillational instability occurs when the transducer velocity amplitude is reduced and the drop equatorial radius becomes less than $2 \mathrm{~mm}$. This observation seems to be in agreement with the results of Lin and Lin, ${ }^{54}$ where they found that the decrease in the sound pressure below a threshold causes a transition from a stable levitation to a self-excited oscillation.

In the results of Figs. 11-13, we have only investigated the position and shape oscillations caused by the oscillation instability, ${ }^{56,}$
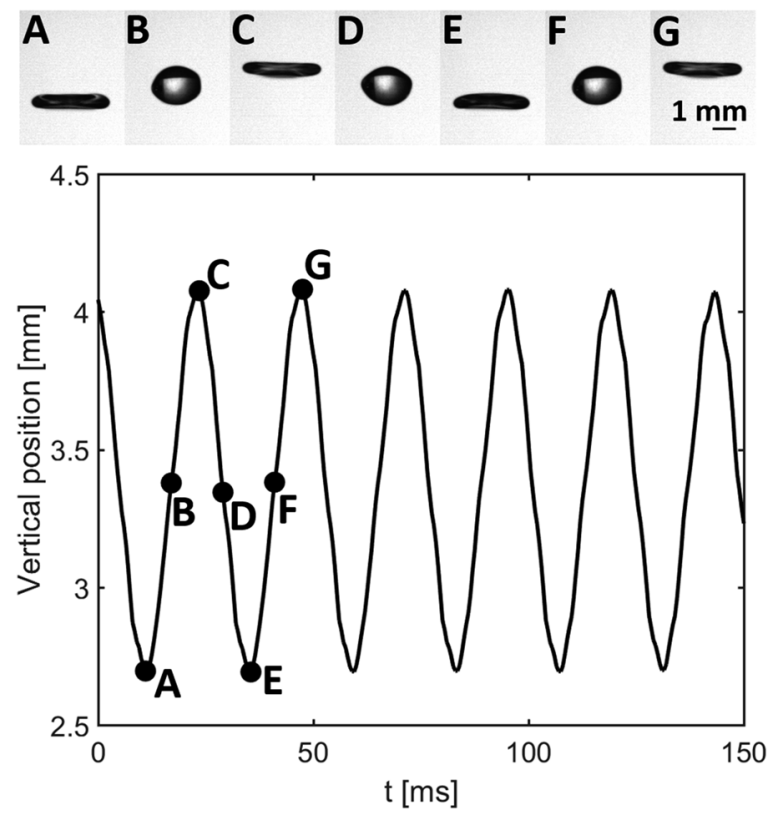

FIG. 13. Vertical and shape oscillation of a water drop of $1 \mathrm{~mm}$ equivalent spherical radius over time. The levitator is driven above the resonance $(H=7.64 \mathrm{~mm})$. A video showing the coupled vertical and shape oscillation is available online. Multimedia view: https://doi.org/10.1063/1.5121728.8 
which only occurs when the transducer-reflector distance is greater than the resonance distance. It is worth mentioning that oscillations of the drop can also be caused by the acoustic streaming. ${ }^{37,58-60}$ As discussed recently, ${ }^{73}$ when the drop radius decreases and becomes closer to the thickness of the acoustic boundary layer $\left(\sqrt{2 \eta / \rho_{0} \omega}\right)$, where $\eta$ is the dynamic viscosity of the air, the influence of the acoustic streaming increases, leading to undesired oscillations of the levitating drop. This is particularly important for drops of less than $100 \mu \mathrm{m}$ in diameter, in which the drag force caused by the streaming can exceed the acoustic radiation force and eject the drop from the levitator. Although the streaming affects the levitation stability, it can be useful in certain types of X-ray diffraction experiments ${ }^{33}$ or for improving the mixing performance inside the drop. ${ }^{39}$ Despite its importance in acoustic levitation, the analysis of the acoustic streaming is beyond the scope of this paper in which the drops were at least 10 times larger than $100 \mu \mathrm{m}$.

\section{CONCLUSIONS}

We have investigated the acoustic levitation stability of water drops in a resonant single-axis acoustic levitator. A numerical model combining the finite element method with the YoungLaplace equation was used to simulate the shape of a levitating drop when the levitator operates above and below resonance. Experiments with a high-speed camera were also carried out to investigate the static and the dynamic behavior of levitating water drops. This process was done systematically using an experimental setup composed of a translation stage that moves the emitter and a scale that measures the radiation force on the reflector.

The numerical and experimental results indicate that if the emitter-reflector separation is below the resonant distance, water drops disintegrate when they exceed a certain aspect ratio. In contrast, when the separation is adjusted above resonance, a drop can be drastically flattened into a pancake shape without bursting. This difference in the levitation behavior is explained by the resonance shift effect. Below the resonance distance, the resonance shift effect causes an increase in the acoustic pressure amplitude when the drop is flattened. When the aspect ratio exceeds a certain threshold value, there is a positive feedback loop in which a small increase in the pressure amplitude produces a further flattening of the drop, causing the rapid expansion of the drop equatorial radius. For the levitator operating above resonance, the resonance shift effect causes a decrease in the pressure amplitude when the drop becomes more flattened. Consequently, when the drop is substantially flattened, an increase in the transducer velocity amplitude causes a decrease in the acoustic pressure amplitude, which allows the drop to be flattened into a pancake shape.

Experiments with the high-speed camera reveal that the drop disintegration mechanism depends on whether the levitator operates below or above the resonance. Below the resonance, the drop equatorial radius increases exponentially with time after the aspect ratio reaches a threshold value. For the levitator operating above the resonance, the drop disintegration involves the following sequence of events: (1) drop flattening into a pancake shape, (2) growing of capillary waves on the central surface of the drop, and (3) atomization into very small droplets.
Our simulation results also provide a possible explanation for the inability to levitate nonsmall drops when the levitator operates below the resonance. Similar to the disintegration mechanism caused by the resonance shift effect, our simulation indicates that when the drop size exceeds a certain limit, the pressure amplitude increases exponentially with time causing the drop disintegration. This effect only occurs if the levitator operates below the resonance, which means that larger drops can be levitated when the levitator operates above the resonance.

We observed experimentally that when the levitator operates above the resonance, the drops are subjected to oscillational instability which causes vertical oscillations or shape oscillations of the liquid drops. It was also observed that a stable levitation can be achieved above the resonance if the transducer velocity amplitude is increased so that the drop becomes substantially flattened.

\section{ACKNOWLEDGMENTS}

This work was supported by the São Paulo Research Foundation-FAPESP (Grant No. 2017/27078-0).

\section{REFERENCES}

${ }^{1}$ S. Santesson and S. Nilsson, "Airborne chemistry: Acoustic levitation in chemical analysis,” Anal. Bioanal. Chem. 378, 1704 (2004).

${ }^{2}$ R. Tuckermann, L. Puskar, M. Zavabeti, R. Sekine, and D. McNaughton, "Chemical analysis of acoustically levitated drops by Raman spectroscopy," Anal. Bioanal. Chem. 394, 1433 (2009).

${ }^{3}$ C. J. Benmore and J. K. R. Weber, "Amorphization of molecular liquids of pharmaceutical drugs by acoustic levitation,” Phys. Rev. X 1, 011004 (2011).

${ }^{4}$ A. Scheeline and R. L. Behrens, "Potential of levitated drops to serve as microreactors for biophysical measurements," Biophys. Chem. 165-166, 1 (2012).

${ }^{5}$ S. K. Cho, H. Moon, and C. J. Kim, "Creating, transporting, cutting, and merging liquid droplets by electrowetting-based actuation for digital microfluidic circuits," J. Microelectromech. Syst. 12, 70 (2003).

${ }^{6}$ K. Choi, A. H. C. Ng, R. Fobel, and A. R. Wheeler, "Digital microfluidics," Annu. Rev. Anal. Chem. 5, 413 (2012).

${ }^{7}$ S. Santra, S. Mandal, and S. Chakraborty, "Electrohydrodynamics of confined two-dimensional liquid droplets in uniform electric field," Phys. Fluids 30, 062003 (2018).

${ }^{8}$ S. Santra, D. Sen, S. Das, and S. Chakraborty, "Electrohydrodynamic interaction between droplet pairs in a confined shear flow," Phys. Fluids 31, 032005 (2019).

${ }^{9}$ K. Zhang, Q. Liang, S. Ma, X. Mu, P. Hu, Y. Wang, and G. Luo, “On-chip manipulation of continuous picoliter-volume superparamagnetic droplets using a magnetic force," Lab Chip 9, 2992 (2009).

${ }^{10}$ M. Qiu, S. Afkhami, C. Y. Chen, and J. J. Feng, "Interaction of a pair of ferrofluid drops in a rotating magnetic field," J. Fluid Mech. 846, 121 (2018).

${ }^{11}$ S. Afkhami, A. J. Tyler, Y. Renardy, M. Renardy, T. G. St. Pierre, R. C. Woodward, and J. S. Riffle, "Deformation of a hydrophobic ferrofluid droplet suspended in a viscous medium under uniform magnetic fields," J. Fluid Mech. 663, 358 (2010).

${ }^{12}$ M. L. Cordero, D. R. Burnham, C. N. Baroud, and D. McGloin, “Thermocapillary manipulation of droplets using holographic beam shaping: Microfluidic pin ball,” Appl. Phys. Lett. 93, 034107 (2008).

${ }^{13}$ C. N. Baroud, J. P. Delville, F. Gallaire, and R. Wunenburger, "Thermocapillary valve for droplet production and sorting," Phys. Rev. E 75, 046302 (2007).

${ }^{14}$ D. Foresti, M. Nabavi, M. Klingauf, A. Ferrari, and D. Poulikakos, "Acoustophoretic contactless transport and handling of matter in air," Proc. Natl. Acad. Sci. U. S. A. 110, 12549 (2013).

${ }^{15}$ S. P. Zhang, J. Lata, C. Chen, J. Mai, F. Guo, Z. Tian, L. Ren, Z. Mao, P. H. Huang, P. Li, S. Yang, and T. J. Huang, "Digital acoustofluidics enables contactless and programmable liquid handling," Nat. Commun. 9, 2928 (2018). 
${ }^{16}$ A. Ozcelik, J. Rufo, F. Guo, Y. Gu, P. Li, J. Lata, and T. J. Huang, "Acoustic tweezers for the life sciences," Nat. Methods 15, 1021 (2018).

${ }^{17}$ E. H. Brandt, "Acoustic physics. Suspended by sound," Nature 413, 474 (2001).

${ }^{18} \mathrm{M}$. A. B. Andrade, N. Pérez, and J. C. Adamowski, "Review of progress in acoustic levitation," Braz. J. Phys. 48, 190 (2018).

${ }^{19}$ L. Meng, F. Cai, F. Li, W. Zhou, L. Niu, and H. Zheng, "Acoustic tweezers," J. Phys. D: Appl. Phys. 52, 273001 (2019).

${ }^{20}$ R. H. Morris, E. R. Dye, P. Docker, and M. I. Newton, "Beyond the Langevin horn: Transducer arrays for the acoustic levitation of liquid drops," Phys. Fluids 31, 101301 (2019).

${ }^{21} \mathrm{H}$. Bruus, "Acoustofluidics 7: The acoustic radiation force on small particles," Lab Chip 12, 1014 (2012).

${ }^{22} \mathrm{E}$. H. Trinh, "Compact acoustic levitation device for studies in fluid dynamics and material science in the laboratory and microgravity," Rev. Sci. Instrum. 56, 2059 (1985).

${ }^{23}$ W. J. Xie and B. Wei, "Parametric study of single-axis acoustic levitation," Appl. Phys. Lett. 79, 881 (2001).

${ }^{24}$ J. K. R. Weber, C. A. Rey, J. Neuefeind, and C. J. Benmore, "Acoustic levitator for structure measurements on low temperature liquid droplets," Rev. Sci. Instrum. 80, 083904 (2009).

${ }^{25}$ D. Koyama and K. Nakamura, "Noncontact ultrasonic transportation of small objects over long distances in air using a bending vibrator and a reflector," IEEE Trans. Ultrason. Ferroelectr. Freq. Control 57, 1152 (2010).

${ }^{26}$ A. Marzo, A. Barnes, and B. W. Drinkwater, "TinyLev: A multi-emitter singleaxis acoustic levitator," Rev. Sci. Instrum. 88, 085105 (2017).

${ }^{27}$ R. Kashima, D. Koyama, and M. Matsukawa, "Two-dimensional noncontact transportation of small objects in air using flexural vibration of a plate," IEEE Trans. Ultrason. Ferroelectr. Freq. Control 62, 2161 (2015).

${ }^{28}$ Y. Ochiai, T. Hoshi, and J. Rekimoto, "Three-dimensional mid-air acoustic manipulation by ultrasonic phased arrays," PLoS One 9, e97590 (2014).

${ }^{29}$ A. Marzo, S. A. Seah, B. W. Drinkwater, D. R. Sahoo, B. Long, and S. Subramanian, "Holographic acoustic elements for manipulation of levitated objects," Nat. Commun. 6, 8661 (2015).

${ }^{30}$ A. Marzo and B. W. Drinkwater, "Holographic acoustic tweezers," Proc. Natl. Acad. Sci. U. S. A. 116, 84 (2019).

${ }^{31} J$ J. Schenk, L. Tröbs, F. Emmerling, J. Kneipp, U. Panne, and M. Albrecht, "Simultaneous UV/Vis spectroscopy and surface enhanced Raman scattering of nanoparticle formation and aggregation in levitated droplets," Anal. Methods $\mathbf{4}$, 1252 (2012)

${ }^{32}$ L. Puskar, R. Tuckermann, T. Frosch, J. Popp, V. Ly, D. McNaughton, and B. R. Wood, "Raman acoustic levitation spectroscopy of red blood cells and Plasmodium falciparum trophozoites," Lab Chip 7, 1125 (2007).

${ }^{33} \mathrm{~S}$. Tsujino and T. Tomizaki, "Ultrasonic acoustic levitation for fast frame rate X-ray protein crystallography at room temperature," Sci. Rep. 6, 25558 (2016).

${ }^{34}$ J. Leiterer, F. Delißen, F. Emmerling, A. F. Thünemann, and U. Panne, "Structure analysis using acoustically levitated droplets," Anal. Bioanal. Chem. 391, 1221 (2008).

${ }^{35}$ E. H. Trinh, P. L. Marston, and J. L. Robey, "Acoustic measurement of the surface tension of levitated drops," J. Colloid Interface Sci. 124, 95 (1988).

${ }^{36}$ Y. Tian, R. G. Holt, and R. E. Apfel, "A new method for measuring liquid surface tension with acoustic levitation," Rev. Sci. Instrum. 66, 3349 (1995).

${ }^{37}$ K. Kobayashi, A. Goda, K. Hasegawa, and Y. Abe, "Flow structure and evaporation behavior of an acoustically levitated droplet," Phys. Fluids 30, 082105 (2018).

${ }^{38} \mathrm{Y}$. Niimura and K. Hasegawa, "Evaporation of droplet in mid-air: Pure and binary droplets in single-axis acoustic levitator," PLoS One 14, e0212074 (2019).

${ }^{39}$ A. Watanabe, K. Hasegawa, and Y. Abe, "Contactless fluid manipulation in air: Droplet coalescence and active mixing by acoustic levitation," Sci. Rep. 8, 10221 (2018).

${ }^{40}$ M. A. B. Andrade, T. S. A. Camargo, and A. Marzo, "Automatic contactless injection, transportation, merging, and ejection of droplets with a multifocal point acoustic levitator," Rev. Sci. Instrum. 89, 125105 (2018).
${ }^{41}$ P. L. Marston, "Shape oscillation and static deformation of drops and bubbles driven by modulated radiation stresses-Theory," J. Acoust. Soc. Am. 67, 15 (1980).

${ }^{42}$ E. H. Trinh and C. Hsu, "Equilibrium shapes of acoustically levitated drops," J. Acoust. Soc. Am. 79, 1335 (1986).

${ }^{43}$ C. P. Lee, A. V. Anilkumar, and T. G. Wang, "Static shape and instability of an acoustically levitated liquid drop," Phys. Fluids A 3, 2497 (1991).

${ }^{44}$ Y. Tian, R. G. Holt, and R. E. Apfel, "Deformation and location of an acoustically levitated liquid drop," J. Acoust. Soc. Am. 93, 3096 (1993).

${ }^{45}$ C. P. Lee, A. V. Anilkumar, and T. G. Wang, "Static shape of an acoustically levitated drop with wave-drop interaction," Phys. Fluids 6, 3554 (1994).

${ }^{46}$ A. L. Yarin, M. Pfaffenlehner, and C. Tropea, "On the acoustic levitation of droplets," J. Fluid Mech. 356, 65 (1998).

${ }^{47}$ W. J. Xie and B. Wei, "Dynamics of acoustically levitated disk samples," Phys. Rev. E 70, 046611 (2004).

${ }^{48}$ E. G. Lierke, D. Lühmann, and E. W. Leung, "Terrestrial levitation, deformation and disintegration (atomization) of liquids and melts in a one-axial acoustic standing wave," AIP Conf. Proc. 197, 71-80 (1990).

${ }^{49}$ A. V. Anilkumar, C. P. Lee, and T. G. Wang, "Stability of an acoustically levitated and flattened drop: An experimental study," Phys. Fluids 5, 2763 (1993).

${ }^{50}$ W. T. Shi, R. E. Apfel, and R. G. Holt, "Instability of a deformed liquid drop in an acoustic field," Phys. Fluids 7, 2601 (1995).

${ }^{51}$ Z. C. Feng and Y. H. Su, "Numerical simulations of the translational and shape oscillations of a liquid drop in an acoustic field," Phys. Fluids 9, 519 (1997).

${ }^{52}$ A. L. Yarin, D. A. Weiss, G. Brenn, and D. Rensink, "Acoustically levitated drops: Drop oscillation and break-up driven by ultrasound modulation," Int. J. Multiphase Flow 28, 887 (2002).

${ }^{53}$ C. L. Shen, W. J. Xie, and B. Wei, "Parametrically excited sectorial oscillation of liquid drops floating in ultrasound," Phys. Rev. E 81, 046305 (2010).

${ }^{54}$ P. C. Lin and I. Lin, "Acoustically levitated dancing drops: Self-excited oscillation to chaotic shedding," Phys. Rev. E 93, 021101 (2016).

${ }^{55} \mathrm{E}$. Bänsch and M. Götz, "Numerical study of droplet evaporation in an acoustic levitator,” Phys. Fluids 30, 037103 (2018).

${ }^{56} \mathrm{~J}$. Rudnick and M. Barmatz, "Oscillational instabilities in single-mode acoustic levitators," J. Acoust. Soc. Am. 87, 81 (1990).

${ }^{57}$ M. A. B. Andrade, S. Polychronopoulos, G. Memoli, and A. Marzo, "Experimental investigation of the particle oscillation instability in a single-axis acoustic levitator," AIP Adv. 9, 035020 (2019).

${ }^{58}$ E. H. Trinh and J. L. Robey, "Experimental study of streaming flows associated with ultrasonic levitators," Phys. Fluids 6, 3567 (1994).

${ }^{59}$ Y. Abe, D. Hyuga, S. Yamada, and K. Aoki, "Study on internal flow and surface deformation of large droplet levitated by ultrasonic wave," Ann. N. Y. Acad. Sci. 1077, 49 (2006)

${ }^{60} \mathrm{~A}$. Rednikov and N. Riley, "A simulation of streaming flows associated with acoustic levitators," Phys. Fluids 14, 1502 (2002).

${ }^{61}$ Y. A. Ilinskii, B. Lipkens, T. S. Lucas, T. W. Van Doren, and E. A. Zabolotskaya, "Nonlinear standing waves in an acoustical resonator," J. Acoust. Soc. Am. 104, 2664 (1998).

${ }^{62}$ M. A. B. Andrade, T. S. Ramos, F. T. A. Okina, and J. C. Adamowski, "Nonlinear characterization of a single-axis acoustic levitator," Rev. Sci. Instrum. 85, 045125 (2014).

${ }^{63}$ R. T. Beyer, "Radiation pressure-The history of a mislabled tensor," J. Acoust. Soc. Am. 63, 1025 (1978).

${ }^{64}$ Z. Y. Hong, W. Zhai, N. Yan, and B. Wei, "Measurement and simulation of acoustic radiation force on a planar reflector," J. Acoust. Soc. Am. 135, 2553 (2014).

${ }^{65} \mathrm{M}$. Barmatz and P. Collas, "Acoustic radiation potential on a sphere in plane, cylindrical, and spherical standing wave fields," J. Acoust. Soc. Am. 77, 928 (1985).

${ }^{66}$ E. Leung, C. P. Lee, N. Jacobi, and T. G. Wang, "Resonance frequency shift of an acoustic chamber containing a rigid sphere," J. Acoust. Soc. Am. 72, 615 (1982).

${ }^{67}$ S. D. Danilov and M. A. Mironov, "Breakup of a droplet in a high-intensity sound field," J. Acoust. Soc. Am. 92, 2747 (1992). 
${ }^{68}$ B. Pathak and S. Basu, "Deformation pathways and breakup modes in acoustically levitated bicomponent droplets under external heating," Phys. Rev. E 93, 033103 (2016)

${ }^{69}$ D. Zang, L. Li, W. Di, Z. Zhang, C. Ding, Z. Chen, W. Shen, B. P. Binks, and $\mathrm{X}$. Geng, "Inducing drop to bubble transformation via resonance in ultrasound," Nat. Commun. 9, 3546 (2018).

${ }^{70}$ W. Di, Z. Zhang, L. Li, K. Lin, J. Li, X. Li, B. P. Binks, X. Chen, and D. Zang, "Shape evolution and bubble formation of acoustically levitated drops," Phys. Rev. Fluids 3, 103606 (2018).
${ }^{71}$ L. D. Landau and E. M. Lifshitz, Fluid Mechanics, Landau and Lifshitz, Course of Theoretical Physics (Pergamon Press, Oxford, 1987).

${ }^{72}$ D. L. Geng, W. J. Xie, N. Yan, and B. Wei, "Vertical vibration and shape oscillation of acoustically levitated water drops," Appl. Phys. Lett. 105, 104101 (2014).

${ }^{73}$ S. Tsujino, Y. Sato, Y. Takeda, and T. Tomizaki, "Oscillation resonances and anisotropic damping of the motion of acoustically levitated droplets in single-axis acoustic levitators," Appl. Phys. Lett. 115, 053702 (2019). 